\title{
Comparative Effectiveness of Abdominal versus Laparoscopic Radical Hysterectomy for Cervical Cancer in the Postdissemination Era
}

\author{
Jin Hee Kim, $P h D^{1}$ \\ Kyungjoo Kim, MS ${ }^{2}$ \\ Seo Jin Park, MS ${ }^{1}$ \\ Jung-Yun Lee, $M D, P h D^{3}$ \\ Kidong Kim, MD, $\mathrm{PhD}^{4}$ \\ Myong Cheol Lim, MD, PhD $5,6,7,8$ \\ Jae Weon Kim, MD, $P h D^{9}$
}

\begin{abstract}
Purpose
Despite the benefits of minimally invasive surgery for cervical cancer, there are a lack of randomized trials comparing laparoscopic radical hysterectomy and abdominal radical hysterectomy. We compared morbidity, cost of care, and survival between abdominal radical hysterectomy and laparoscopic radical hysterectomy for cervical cancer.
\end{abstract}

\section{Materials and Methods}

We used the Korean nationwide database to identify women with cervical cancer who underwent radical hysterectomy from January 1, 2011 to December 31, 2014. Patients who underwent abdominal radical hysterectomy were compared to those who underwent laparoscopic radical hysterectomy. Perioperative morbidity, the use of adjuvant therapy, and survival were evaluated after propensity score balancing.

\section{Results}

We identified 6,335 patients, including 3,235 who underwent abdominal radical hysterectomy and 3,100 who underwent laparoscopic radical hysterectomy. The use of laparoscopic radical hysterectomy increased from $46.1 \%$ in 2011 to $51.8 \%$ in 2014 . Patients who were younger, had a more recent year of diagnosis, and were treated in the metropolitan area were more likely to undergo a laparoscopic procedure $(p<0.001)$. Compared to abdominal radical hysterectomy, laparoscopic radical hysterectomy was associated with lower rates of complication, fewer transfusions, a shorter hospital stay, less adjuvant therapy, and reduced total medical costs $(p<0.001)$. Laparoscopic surgery was associated with a better overall survival than abdominal operation (hazard ratio, 0.74 ; 95\% confidence interval, 0.64 to 0.85).

\section{Conclusion}

In the postdissemination era, laparoscopic radical hysterectomy was associated with more favorable morbidity profiles, a lower cost of care, and comparable survival than abdominal radical hysterectomy.
Correspondence: Jung-Yun Lee, MD, PhD Department of Obstetrics and Gynecology, Institute of Women's Life Medical Science, Yonsei University College of Medicine, 50-1 Yonsei-ro, Seodaemun-gu, Seoul 03722, Korea Tel: 82-2-2228-2237

Fax: 82-2-313-8357

E-mail: jungyunlee@yuhs.ac

Received February 20, 2018

Accepted September 10, 2018

Published Online September 11, 2018

\section{Key words}

Uterine cervical neoplasms, Radical hysterectomy, Minimally invasive surgery, Laparoscopy 


\section{Introduction}

Originally described by Wertheim in the 1890s and subsequently re-popularized by Meigs in the 1950s, radical hysterectomy has long been established as a standard treatment for early-stage cervical cancer [1]. Although abdominal radical hysterectomy represented the standard surgical approach for many years, minimally invasive approaches have been widely adopted in gynecologic oncology over the past few decades $[2,3]$.

Radical hysterectomy and pelvic lymphadenectomy are standard surgical treatments for early-stage cervical cancer. Despite the excellent 5-year overall survival rate $[4,5]$, the surgical treatment resulted in substantial long-term morbidity, such as lower urinary track dysfunction, sexual dysfunction, and colorectal motility disorders [6-8]. Furthermore, the surgical approach was traditionally performed through laparotomy and associated with a high incidence of perioperative morbidity and mortality.

Laparoscopic radical hysterectomy was introduced to decrease the morbidity of the operative procedure. Numerous retrospective studies have compared laparoscopic and abdominal radical hysterectomy for cervical cancer, and showed that the laparoscopic approach is oncologically safe and associated with fewer postoperative complications and earlier recovery [9-16]. However, there have been concerns about the oncologic safety of laparoscopic radical hysterectomy due to difficulties in achieving a sufficient resection margin from the complex procedure itself. However, patient acceptance of minimally invasive surgery increases even in the absence of randomized phase III trials that show advantages to these procedures [17]. Currently, only one randomized clinical trial is evaluating laparoscopic or robotic radical hysterectomy with abdominal radical hysterectomy in patients with early-stage cervical cancer [18].

Despite the common use of minimally invasive surgery operation for cervical cancer, there are no population-level data evaluating survival after the procedure in unselected patients. We performed a population-based analysis to compare the effectiveness between abdominal radical hysterectomy and laparoscopic radical hysterectomy for cervical cancer.

\section{Materials and Methods}

\section{Data source}

The Korean Health Insurance Review and Assessment Service (HIRA) database was used for the analysis. HIRA is an independent body established to review the claims data and assess the quality of health care. In Korea, since health insurance is applied to all citizens and it is a fee-for-service system, everyone's health information is coded and registered in the HIRA database. Therefore, this database captures inpatient and outpatient data on disease and services (procedures and operations) in a timely manner. Disease codes used in the HIRA database are standardized according to the Korean Classification of Disease, sixth version, which follows the International Classification of Diseases, 10th edition (ICD-10) [19]. Procedure and operation codes use HIRA's own code and are released yearly [20].

\section{Cohort selection}

Women 18 years and older who underwent radical hysterectomy for cervical cancer were identified from the HIRA database between January 1, 2011 and December 31, 2014. A multidisciplinary panel consisting of gynecologic oncologists (J.Y.L., K.K., M.C.L., and J.W.K.) and epidemiologists (J.H.K., K.K., and S.J.P.) identified the patients with newly diagnosed cervical cancer. Disagreements were resolved by consensus after discussion. Newly diagnosed cervical cancer in a patient with radical hysterectomy was defined by using the following criteria: the presence of an ICD-10 code for cervical cancer and HIRA operation codes for radical hysterectomy. ICD-10 codes for cervical cancer were C530, C531, C538, and C539. HIRA operation codes for radical hysterectomy were R4154 and R4155.

Patients were stratified into two groups based on the type of radical hysterectomy performed: abdominal or laparoscopic. To identify women who underwent the laparoscopic procedure, the database was searched to identify charges for laparoscopic materials (N0031001). In Korea, insurance does not cover the cost of robotic operation. Therefore, robotic operation could not be identified in this database.

\section{Characteristics}

Patient characteristics analyzed included age at operation, year of diagnosis, insurance status (Medicare or Medicaid), comorbidities (Charlson comorbidity index [21] score), and the extent of lymphadenectomy. Hospital characteristics analyzed included the hospital location (metropolitan or nonmetropolitan), hospital region (metropolitan area [Seoul/ 
Gyeonggi Province], Middle [Chungcheong Province], East [Gangwon Province], South West [Jeolla Province], and South East [Gyeongsang Province]), and hospital size (tertiary general hospital, general hospital, hospital, or clinic).

\section{Outcomes}

The primary outcome was overall survival. Death was deemed to have occurred when patients did not use any medical service for 12 consecutive months after discharge. Additionally, perioperative morbidity and mortality were analyzed. Perioperative mortality was defined as in-hospital death. Perioperative morbidity and the use of blood transfusions were recorded. We used methods in previous studies in obtaining morbidity and survival data extraction from HIRA database [22,23]. To identify complications, diagnostic and procedure codes were used (S1 Table). Perioperative morbidity was classified into the following categories: (1) intraoperative complications (bladder injury, ureter injury, intestinal injury, and vascular injury); (2) postoperative complications (bladder dysfunction, lymphocele, lymphedema, bowel obstruction, and fistula); (3) surgical site complications (wound abscess, disruption, and incisional hernia); (4) medical complications (venous thromboembolism, myocardial infarction, cardiopulmonary arrest, respiratory failure, renal failure, bacteremia / sepsis, shock, pneumonia, delirium, acute pyelonephritis, and stroke). Blood transfusions during hospitalization for the operation were counted. For intraoperative complications among perioperative morbidities, the event that occurred during hospitalization for the operation was considered, whereas for postoperative complications, surgical site complications, medical complication, and events that occurred within 6 months postoperatively were considered. The aforementioned complications were assessed using the 6th revision of the Korean Standard Classification of Diseases based on ICD-10 and current procedural terminology codes (S1 Table).

The use of adjuvant vaginal brachytherapy, whole pelvic radiation, and chemotherapy during the 3 months postoperatively was noted for each patient.

The HIRA database includes an itemized, data-stamped $\log$ of all items billed to a patient, including drugs, laboratory and radiologic tests, and therapeutic services. Medical costs per person during hospitalization for the operation within 30 days, 90 days, and 6 months postoperatively were evaluated. This analysis did not consider uninsured benefits. All costs were reported in December 31, 2014 in United States (US) dollars with an exchange rate of 1 US dollar equaling 1,107 Korean won. Costs are reported as medians and interquartile ranges. This study also analyzed use of the intensive care unit service, 30-day reoperation, non-routine discharge, 30-day readmission, 30-day emergency room visit, and leng- th of stay for the operation by capitalizing on the HIRA database.

\section{Statistical analysis}

Frequency distributions between categorical variables were compared using the chi-square test, and continuous variables were compared with the Student $t$ test. The multivariable logistic regression that included patients and hospital characteristics was used to identify the factors that affect the use of laparoscopic radical hysterectomy.

The inverse probability of treatment weighting (IPTW) method based on the propensity score was applied to balance the observed confounders between the methods of operation. With this technique, weights for patients who underwent laparoscopic radical hysterectomy were the inverse of 1-propensity score, and weights for patients who had abdominal radical hysterectomy were the inverse of the propensity score [24]. The propensity score was the predicted probability of treatment, laparoscopic radical hysterectomy, or abdominal radical hysterectomy, in this analysis $[25,26]$. To calculate the propensity score, we used a logistic regression model that included patient characteristics (age at operation, year of diagnosis, insurance status, comorbidities, and the extent of lymphadenectomy), hospital characteristics (hospital location, hospital region, and hospital size), and two-way interaction terms. The predicted probability was estimated for each patient. After propensity score balancing, subsequent treatment, morbidity, and mortality were compared between the two groups. Overall mortality was evaluated between the two groups using the Kaplan-Meier method and Cox proportional hazard models. All statistical tests were two-sided. A p-value of $<0.05$ was considered statistically significant. To perform all statistical analyses, SAS software ver. 9.1.3 (SAS Institute Inc., Cary, NC) was used.

\section{Ethical statement}

This study was approved by the institutional review board of Yonsei University Health System, Severance Hospital (approval No. 4-2016-0440). As HIRA database are publicly available, written informed consent was waived for this study.

\section{Results}

We identified 6,335 patients, including 3,235 women (51.1\%) who underwent abdominal radical hysterectomy and 3,100 (48.9\%) who underwent laparoscopic radical hysterec- 


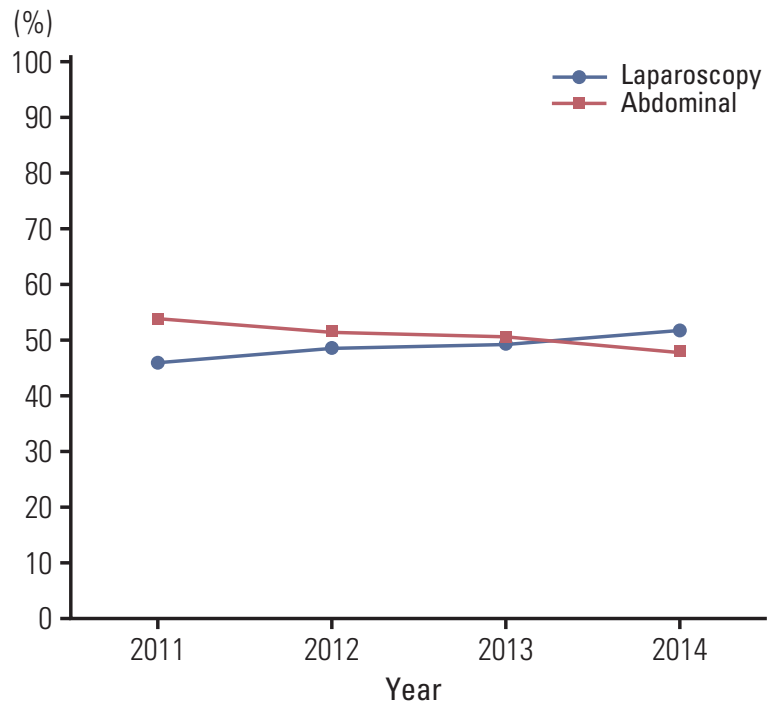

Fig. 1. Trends in the use of laparoscopic and abdominal radical hysterectomy (2011-2014).

tomy. The use of laparoscopic surgery increased with time. The use of laparoscopic radical hysterectomy increased from 46.1\% in 2011 to $51.8 \%$ in 2014 (p for trend=0.016) (Fig. 1). Table 1 shows the characteristics of women who underwent abdominal radical hysterectomy versus laparoscopic radical hysterectomy. When patients were stratified according to the surgical approach, statistically significant differences were observed with respect to age, year of diagnosis, insurance status, comorbidities, the extent of lymphadenectomy, and hospital region. After propensity score balancing, there was no significant difference in these variables between the two groups.

In a multivariable model for identifying predictors of undergoing laparoscopic operation, age was the strongest predictor associated with minimally invasive operation. Patients who were younger, had a more recent year of diagnosis, were hospitalized in a metropolitan area, and had a Medicare insurance status were more likely to undergo laparoscopic radical hysterectomy ( $\mathrm{p}<0.05$ for all) (S2 Table). In contrast, patients with more medical comorbidities who were hospitalized at a small-sized hospital (a hospital or clinic) were less likely to undergo a laparoscopic radical hysterectomy ( $\mathrm{p}<0.05$ for all) (S2 Table).

Laparoscopic radical hysterectomy had favorable morbidity profiles and a health care utilization index compared to abdominal radical hysterectomy (S3 Table). Compared with abdominal radical hysterectomy, laparoscopic radical hysterectomy was associated with fewer intraoperative complications $(9.9 \%$ vs. $12.0 \%, \mathrm{p}<0.001)$, fewer postoperative complications ( $19.5 \%$ vs. $21.7 \%$, $\mathrm{p}=0.002)$, and fewer surgical site complications $(6.2 \%$ vs. $8.1 \%, \mathrm{p}<0.001)$. Regarding the health utilization index, the median length of stay was shorter with laparoscopic radical hysterectomy than with abdominal radical hysterectomy $(\mathrm{p}<0.001)$. When focusing on inpatient outcomes, the median cost of care was higher with laparoscopic radical hysterectomy than with abdominal hysterectomy (US \$4,421 vs. \$4,147), although this difference was not statistically significant. When focusing on postoperative outcomes, patients undergoing laparoscopic radical hysterectomy had lower 30-day reoperation rates. In addition, patients undergoing laparoscopic radical hysterectomy were associated with a lower cost of care within 30 days, 90 days, and 6 months after discharge.

The overall rates of intraoperative complications (odds ratio [OR], $0.73 ; 95 \%$ confidence interval [CI], 0.63 to 0.86 ), surgical site complications (OR, $0.73 ; 95 \% \mathrm{CI}, 0.60$ to 0.88 ), medical complications (OR, $0.88 ; 95 \% \mathrm{CI}, 0.79$ to 0.98$)$, and blood transfusions (OR, $0.28 ; 95 \%$ CI, 0.25 to 0.31 ) were lower with laparoscopic radical hysterectomy than with abdominal radical hysterectomy (Table 2). After propensity score balancing, laparoscopic radical hysterectomy still had lower rates of intraoperative complications $(\mathrm{OR}, 0.80 ; 95 \% \mathrm{CI}, 0.72$ to 0.90$)$, postoperative complications (OR, $0.87 ; 95 \% \mathrm{CI}, 0.80$ to 0.95 ), surgical site complications (OR, $0.75 ; 95 \% \mathrm{CI}, 0.66$ to 0.86 ), and blood transfusions (OR, $0.30 ; 95 \% \mathrm{CI}, 0.28$ to 0.33 ). In addition, the rate of use of adjuvant therapy was lower (OR, $0.63 ; 95 \%$ CI, 0.57 to 0.69$)$ in patients who underwent laparoscopic radical hysterectomy than in those who underwent abdominal radical hysterectomy.

Table 3 demonstrates the cumulative hazards of adverse outcomes in patients in receiving either laparoscopic or abdominal radical hysterectomy using unadjusted and adjusted multivariable analyses. Unadjusted Cox regression models showed that laparoscopic radical hysterectomy was associated with better overall survival (hazard ratio [HR], $0.52 ; 95 \% \mathrm{CI}, 0.43$ to 0.63 ). Additionally, laparoscopic radical hysterectomy was associated with better overall survival when adjusting for IPTW or IPTW and the covariates. When we performed sensitivity analysis for patients who underwent adjuvant therapy and no adjuvant therapy, the laparoscopic radical hysterectomy group had better survival than the abdominal radical hysterectomy group. This finding was pronounced in patients with no adjuvant therapy (HR, 0.52; $95 \%$ CI, 0.41 to 0.66 ) and the benefits of laparoscopy were marginal in patients with adjuvant therapy (HR, 0.85; 95\% CI, 0.72 to 0.99 ). Likewise, survival statistically significantly improved among patients undergoing laparoscopic radical hysterectomy in all Kaplan-Meier analyses (Fig. 2). 
Table 1. Characteristics of the cohort that underwent laparoscopic and abdominal radical hysterectomy

\begin{tabular}{|c|c|c|c|c|c|c|c|}
\hline \multirow{2}{*}{ Characteristic } & \multirow{2}{*}{ Total } & \multicolumn{3}{|c|}{ Unadjusted } & \multicolumn{3}{|c|}{ Inverse probability of treatment weightinga) } \\
\hline & & Abdominal RH & Laparoscopic RH & p-value ${ }^{b)}$ & Abdominal RH & Laparoscopic RH & p-value ${ }^{c}$ \\
\hline Total patients & $6,335(100)$ & $3,235(100)$ & $3,100(100)$ & & $3,171(100)$ & $3,166(100)$ & \\
\hline \multicolumn{8}{|l|}{ Age at operation (yr) } \\
\hline$\leq 39$ & $1,265(20.0)$ & $587(18.1)$ & $678(21.9)$ & $<0.001$ & $617(19.5)$ & $634(20.0)$ & 0.785 \\
\hline $40-49$ & $2,066(32.6)$ & $1,044(32.3)$ & $1,022(33.0)$ & & $1,045(33.0)$ & $1,020(32.2)$ & \\
\hline $50-59$ & $1,732(27.3)$ & $898(27.8)$ & $834(26.9)$ & & $869(27.4)$ & $874(27.6)$ & \\
\hline$\geq 60$ & $1,272(20.1)$ & $706(21.8)$ & $566(18.3)$ & & $640(20.2)$ & $638(20.2)$ & \\
\hline \multicolumn{8}{|l|}{ Year of diagnosis } \\
\hline 2011 & $1,617(25.5)$ & $872(27.0)$ & $745(24.0)$ & 0.016 & $813(25.6)$ & 807 (25.5) & 0.647 \\
\hline 2012 & $1,572(24.8)$ & $806(24.9)$ & $766(24.7)$ & & $777(24.5)$ & $800(25.3)$ & \\
\hline 2013 & $1,648(26.0)$ & $835(25.8)$ & $813(26.2)$ & & $836(26.4)$ & 809 (25.6) & \\
\hline 2014 & $1,498(23.6)$ & $722(22.3)$ & $776(25.0)$ & & $745(23.5)$ & $750(23.7)$ & \\
\hline \multicolumn{8}{|l|}{ Insurance status } \\
\hline Medicare & $6,084(96.0)$ & $3,071(94.9)$ & $3,013(97.2)$ & $<0.001$ & $3,044(96.0)$ & $3,041(96.1)$ & 0.910 \\
\hline Medicaid & $251(4.0)$ & $164(5.1)$ & $87(2.8)$ & & $127(4.0)$ & $125(3.9)$ & \\
\hline \multicolumn{8}{|l|}{$\begin{array}{l}\text { Comorbidities } \\
\text { (CCI score) }\end{array}$} \\
\hline 2 & $1,874(29.6)$ & $922(28.5)$ & $952(30.7)$ & $<0.001$ & $941(29.7)$ & $934(29.5)$ & 0.988 \\
\hline 3 & $1,502(23.7)$ & $703(21.7)$ & $799(25.8)$ & & $749(23.6)$ & $744(23.5)$ & \\
\hline 4 & 775 (12.2) & 364 (11.3) & 411 (13.3) & & $390(12.3)$ & $388(12.3)$ & \\
\hline$\geq 5$ & $2,184(34.5)$ & $1,246(38.5)$ & $938(30.3)$ & & $1,091(34.4)$ & $1,100(34.7)$ & \\
\hline \multicolumn{8}{|l|}{$\begin{array}{l}\text { Para-aortic } \\
\text { lymphadenectomy }\end{array}$} \\
\hline Yes & $2,410(38.0)$ & $1,354(41.9)$ & $1,056(34.1)$ & $<0.001$ & $1,198(37.8)$ & 1,191 (37.6) & 0.827 \\
\hline No & $3,925(62.0)$ & $1,881(58.1)$ & $2,044(65.9)$ & & $1,973(62.2)$ & $1,975(62.4)$ & \\
\hline \multicolumn{8}{|l|}{ Hospital location } \\
\hline Metropolitan & 4,635 (73.2) & $2,365(73.1)$ & $2,270(73.2)$ & 0.915 & $2,304(72.7)$ & $2,294(72.5)$ & 0.803 \\
\hline Nonmetropolitan & $1,700(26.8)$ & $870(26.9)$ & $830(26.8)$ & & $867(27.3)$ & $872(27.5)$ & \\
\hline \multicolumn{8}{|l|}{ Hospital region } \\
\hline Metropolitan area & $4,216(66.6)$ & $1,963(60.7)$ & $2,253(72.7)$ & $<0.001$ & $2,114(66.7)$ & 2,109 (66.6) & 0.869 \\
\hline Middle & $259(4.1)$ & $136(4.2)$ & $123(4.0)$ & & $131(4.1)$ & $133(4.2)$ & \\
\hline East & $57(0.9)$ & 47 (1.5) & $10(0.3)$ & & $28(0.9)$ & $34(1.1)$ & \\
\hline South West & $379(6.0)$ & $226(7.0)$ & $153(4.9)$ & & $191(6.0)$ & $193(6.1)$ & \\
\hline South East & $1,424(22.5)$ & $863(26.7)$ & $561(18.1)$ & & $707(22.3)$ & $697(22.0)$ & \\
\hline \multicolumn{8}{|l|}{ Hospital volume } \\
\hline $\begin{array}{l}\text { Tertiary general } \\
\text { hospital }\end{array}$ & $4,220(66.6)$ & $2,164(66.9)$ & $2,056(66.3)$ & 0.061 & $2,080(65.6)$ & $2,070(65.4)$ & 0.935 \\
\hline General hospital & 2,097 (33.1) & $1,057(32.7)$ & $1,040(33.5)$ & & 1,082 (34.1) & $1,086(34.3)$ & \\
\hline Hospital and clinic & $18(0.3)$ & $14(0.4)$ & $4(0.1)$ & & $9(0.3)$ & $10(0.3)$ & \\
\hline
\end{tabular}

$\mathrm{RH}$, radical hysterectomy; CCI, Charlson comorbidity index. ${ }^{a}$ Frequency numbers were rounded to integers based on weight,

b)The p-values were derived from the chi-square test, ${ }^{c}$ The $\mathrm{p}$-values were derived from weighted survey logistic model.

\section{Discussion}

During the study period, approximately half of all radical hysterectomies performed in Korea were done laparoscopically. Using a cohort of patients treated in the postdissemi- nation era, we showed that laparoscopic radical hysterectomy was associated with lower rates of complications, lower cost of care, and better survival outcomes than abdominal radical hysterectomy.

Despite limited evaluation of the comparative effectiveness between laparoscopic radical hysterectomy and abdominal 
Table 2. Comparison of morbidity between patients who underwent laparoscopic and abdominal radical hysterectomy for cervical cancer

\begin{tabular}{|c|c|c|c|c|}
\hline & Unadjusted & \multirow{2}{*}{ p-value } & Adjusted by IPTW & \multirow{2}{*}{ p-value } \\
\hline & OR $(95 \% \mathrm{CI})$ & & OR $(95 \% \mathrm{CI})$ & \\
\hline Intraoperative complications & $0.73(0.63-0.86)$ & $<0.001$ & $0.80(0.72-0.90)$ & 0.008 \\
\hline Postoperative complications & $0.97(0.87-1.07)$ & 0.525 & $0.87(0.80-0.95)$ & 0.002 \\
\hline Surgical site complications & $0.73(0.60-0.88)$ & 0.001 & $0.75(0.66-0.86)$ & $<0.001$ \\
\hline Medical complications & $0.88(0.79-0.98)$ & 0.018 & $0.99(0.90-1.08)$ & 0.739 \\
\hline Blood transfusions & $0.28(0.25-0.31)$ & $<0.001$ & $0.30(0.28-0.33)$ & $<0.001$ \\
\hline Postoperative adjuvant therapy & $0.59(0.52-0.67)$ & $<0.001$ & $0.63(0.57-0.69)$ & $<0.001$ \\
\hline Radiation only & $0.92(0.76-1.11)$ & 0.387 & $0.87(0.76-0.99)$ & 0.042 \\
\hline Chemotherapy only & $0.48(0.41-0.56)$ & $<0.001$ & $0.55(0.49-0.61)$ & $<0.001$ \\
\hline CCRT & $0.44(0.39-0.49)$ & $<0.001$ & $0.47(0.43-0.51)$ & $<0.001$ \\
\hline
\end{tabular}

IPTW, inverse probability of treatment weighting; OR, odds ratio; CI, confidence interval; CCRT, concurrent chemoradiation.

Table 3. Hazard ratios for clinical outcomes with laparoscopic versus abdominal radical hysterectomy

\begin{tabular}{|c|c|c|c|c|c|c|}
\hline \multirow{2}{*}{ All-cause mortality } & \multicolumn{2}{|c|}{ Unadjusted } & \multicolumn{2}{|c|}{ Adjusted by IPTW } & \multicolumn{2}{|c|}{ Adjusted by IPTW and PoAT } \\
\hline & HR $(95 \% \mathrm{CI})$ & p-value & HR $(95 \% \mathrm{CI})$ & p-value & $\mathrm{HR}(95 \% \mathrm{CI})$ & p-value \\
\hline Total & $0.52(0.43-0.63)$ & $<0.001$ & $0.61(0.53-0.70)$ & $<0.001$ & $0.74(0.64-0.85)$ & $<0.001$ \\
\hline With adjuvant therapy & $0.71(0.56-0.90)$ & 0.005 & $0.85(0.72-0.99)$ & 0.046 & - & - \\
\hline Without adjuvant therapy & $0.48(0.34-0.67)$ & $<0.001$ & $0.52(0.41-0.66)$ & $<0.001$ & - & - \\
\hline
\end{tabular}

IPTW, inverse probability of treatment weighting; PoAT, postoperative adjuvant therapy; HR, hazard ratio; CI, confidence interval.

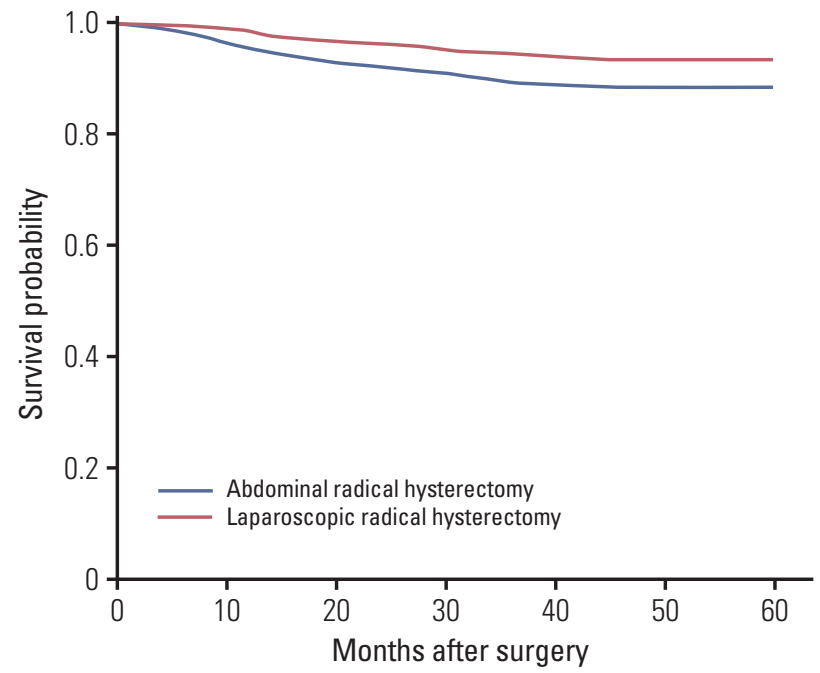

Fig. 2. Overall survival with laparoscopic versus abdominal radical hysterectomy. radical hysterectomy, as well as the absence of randomized trials comparing the two techniques, minimally invasive surgery has been widely used for cervical cancer in the last decade in Korea. In 2011, 46.1\% of radical hysterectomies for cervical cancer were performed minimally invasively. The rate of use of minimally invasive surgery in our study was higher than that in previous studies. Wright et al. [17] reported that only $15 \%$ of patients with cervical cancer underwent laparoscopic or robotic radical hysterectomy during 20062010 from a population-based database. The widespread use of laparoscopy in Korea has been attributed to patients' preference to minimally invasive operation, acceptance of new techniques from gynecologic oncologists, and increasing hospital competition due to patients' demands.

Several of our findings are noteworthy. First, discrepancies were found in the use of laparoscopic surgery in Korea. Not only age, year of diagnosis, and comorbidities but modifiable factors such as one's insurance status and the hospital region affects the use of laparoscopic radical hysterectomy. This finding was in line with that of other studies on hysterectomy for benign and oncologic indications, which showed 
significant disparities in the use of minimally invasive surgery [26].

Second, patients who underwent laparoscopic radical hysterectomy had a lower risk of intraoperative complications, postoperative complications, and surgical site complications even after propensity matching. An important benefit of minimally invasive surgery is lower rates of perioperative morbidity. Numerous prior retrospective studies comparing laparoscopic radical hysterectomy and laparotomy for cervical cancer have consistently shown lower complication rates [9-17]. Most of the studies were based on contemporaneous series of patients from a single institution. Using populationbased database, Wright et al. [17] showed that minimally invasive radical hysterectomy had favorable morbidity profiles with a lower rate of complications than abdominal radical hysterectomy. In this study, only 284 of 1,894 patients (15\%) underwent minimally invasive surgery between 2006 and 2010. In our study, differences in perioperative outcomes became more noticeable with $48.9 \%$ of patients who underwent laparoscopic radical hysterectomy, indicating wide use of minimally invasive surgery.

Third, laparoscopic radical hysterectomy was associated with a lower total cost of care within 6 months postoperatively than open radical hysterectomy. While decision analyses for radical hysterectomy are lacking, a modeling study of simple hysterectomy for endometrial cancer noted that the laparoscopic approach was the least expensive approach from a societal perspective followed by robotic and then abdominal hysterectomy [27]. These findings might result from favorable operative outcomes, such as lower complication rates, a shorter hospital stay, and fewer blood transfusions, of laparoscopic surgery. In addition, one possible explanation for these observations may reside in the characteristics of the population evaluated. The age at operation, comorbidities, and Medicare insurance status represent independent predictors of complications and use of the health care expenditure. Differences in patients' characteristics might affect these results.

Lastly, we found favorable outcomes in survival for laparoscopic radical hysterectomy compared to abdominal radical hysterectomy. Previous retrospective studies have shown non-inferior survival associated with laparoscopic operation compared with laparotomy $[9,10,12,15]$. However, these results were derived from experienced surgeons with a limited number of patients. In the Laparoscopic Approach to Cervical Cancer trial, 740 patients with cervical cancer and tumors less than $4 \mathrm{~cm}$ were randomly assigned to either minimally invasive surgery or laparotomy. Although this study started in January 2008, it is still recruiting participants. The slow recruitment for this study might be due to rarity of the tumor in western countries and patients' reluctance to participate in a study if they prefer minimally invasive opera- tion. Therefore, large observational studies are needed to evaluate survival outcomes.

Encouragingly, we found better survival outcomes with laparoscopic radical hysterectomy than with abdominal radical hysterectomy, before propensity matching. As laparoscopic radical hysterectomy was associated with a lower risk of complications, this benefit may translate to a decrease in all-cause mortality. The incidence of serious complications, such as bowel obstruction, cardiopulmonary arrest, renal failure, and sepsis, was significantly lower with laparoscopic radical hysterectomy than with abdominal radical hysterectomy. This should be emphasized in clinical practice. However, selection bias in the choice of surgical procedure might affect the results. As adjuvant therapy is usually performed based on pathologic findings after radical hysterectomy, we found that adjuvant therapy, radiation and chemotherapy in women who underwent laparoscopic radical hysterectomy, was used less frequently. This suggests that surgeons tend to choose laparoscopic radical hysterectomy for small-volume tumors and select abdominal radical hysterectomy for large-volume tumors. Therefore, we tried to balance the two groups with propensity matching and adjusting adjuvant therapy, and we found the same results of better survival with laparoscopic radical hysterectomy than with abdominal radical hysterectomy. In addition, when we performed sensitivity analysis in patients who underwent adjuvant therapy and no adjuvant therapy, laparoscopic radical hysterectomy was associated with better survival.

Although we included a large cohort of patients, we recognize several limitations. First, we lacked data on tumor characteristics such as the tumor size, stage, histology, and margin status. Differences in tumor characteristics may not only have led to selection bias in the choice of procedure, but also may have influenced procedure-associated morbidity. Although we tried to balance confounders between treatments with propensity score matching, differences in adjuvant treatment still exists. Therefore, we did subgroup analysis according to adjuvant treatment. Second, as with any study of administrative data, we cannot capture individual patient and physician preferences that undoubtedly influenced the treatment decision-making. Third, claims data undercapture complications. Therefore, we categorized complications and tried to find events, using diagnostic codes and procedure codes. Lastly, the HIRA database was not intended to identify survival outcomes, so we could not obtain recurrence-free survival and determine death indirectly by relying on disqualification of the insurance status.

Two studies presented at the March 2018 Society of Gynecologic Oncology Annual Meeting on Women's Cancer, women with early cervical cancer treated by minimally invasive radical hysterectomy were shown to have a significantly higher risk of disease recurrence and poorer long-term sur- 
vival than those of women treated by open surgery [28,29]. Our results were opposite those mentioned above. Some criticisms were raised for the results of Laparoscopic Approach to Carcinoma of the Cervix (LACC) trial [30-32], we should wait for final results coming at least next 2 years. As clinicians confused to abandon laparoscopic surgery for earlystage cervical cancer, our results might be discussed with patients scheduled to undergo radical hysterectomy in Korea.

In summary, laparoscopic radical hysterectomy was associated with a lower risk of perioperative complications and has comparable outcomes with abdominal radical hysterectomy. However, the use of laparoscopic radical hysterectomy for cervical cancer has slowly increased since 2011 and now accounts for more than $50 \%$ of operations for this disease. Increasing the use of laparoscopic surgery in clinical practice may be an important and underused strategy to improve cer- vical cancer outcomes.

\section{Electronic Supplementary Material}

Supplementary materials are available at Cancer Research and Treatment website (https: // www.e-crt.org).

\section{Conflicts of Interest}

Conflict of interest relevant to this article was not reported.

\section{Acknowledgments}

This study was supported by Bumsuk Academic Research Fund in 2016.

\section{References}

1. Dursun P, Gultekin M, Ayhan A. The history of radical hysterectomy. J Low Genit Tract Dis. 2011;15:235-45.

2. Conrad LB, Ramirez PT, Burke W, Naumann RW, Ring KL, Munsell MF, et al. Role of minimally invasive surgery in gynecologic oncology: an updated survey of members of the Society of Gynecologic Oncology. Int J Gynecol Cancer. 2015; 25:1121-7.

3. Lee JY, Kim K, Lee TS, Kang S, Seong SJ, Kim JW, et al. Controversies in the management of endometrial cancer: a survey of the Korean Gynecologic Oncology Group. J Gynecol Oncol. 2015;26:277-83.

4. Jung KW, Won YJ, Kong HJ, Oh CM, Shin A, Lee JS. Survival of korean adult cancer patients by stage at diagnosis, 20062010: national cancer registry study. Cancer Res Treat. 2013;45: 162-71.

5. Siegel R, Naishadham D, Jemal A. Cancer statistics, 2012. CA Cancer J Clin. 2012;62:10-29.

6. Bergmark K, Avall-Lundqvist E, Dickman PW, Henningsohn L, Steineck G. Vaginal changes and sexuality in women with a history of cervical cancer. N Engl J Med. 1999;340:1383-9.

7. Scotti RJ, Bergman A, Bhatia NN, Ostergard DR. Urodynamic changes in urethrovesical function after radical hysterectomy. Obstet Gynecol. 1986;68:111-20.

8. Sood AK, Nygaard I, Shahin MS, Sorosky JI, Lutgendorf SK, Rao SS. Anorectal dysfunction after surgical treatment for cervical cancer. J Am Coll Surg. 2002;195:513-9.

9. Nam JH, Park JY, Kim DY, Kim JH, Kim YM, Kim YT. Laparoscopic versus open radical hysterectomy in early-stage cervical cancer: long-term survival outcomes in a matched cohort study. Ann Oncol. 2012;23:903-11.

10. Park JY, Kim DY, Kim JH, Kim YM, Kim YT, Nam JH. Laparoscopic versus open radical hysterectomy in patients with stage
IB2 and IIA2 cervical cancer. J Surg Oncol. 2013;108:63-9.

11. Abu-Rustum NR, Gemignani ML, Moore K, Sonoda Y, Venkatraman E, Brown C, et al. Total laparoscopic radical hysterectomy with pelvic lymphadenectomy using the argon-beam coagulator: pilot data and comparison to laparotomy. Gynecol Oncol. 2003;91:402-9.

12. Bogani G, Cromi A, Uccella S, Serati M, Casarin J, Pinelli C, et al. Laparoscopic versus open abdominal management of cervical cancer: long-term results from a propensity-matched analysis. J Minim Invasive Gynecol. 2014;21:857-62.

13. Frumovitz M, dos Reis R, Sun CC, Milam MR, Bevers MW, Brown J, et al. Comparison of total laparoscopic and abdominal radical hysterectomy for patients with early-stage cervical cancer. Obstet Gynecol. 2007;110:96-102.

14. Magrina JF, Kho RM, Weaver AL, Montero RP, Magtibay PM. Robotic radical hysterectomy: comparison with laparoscopy and laparotomy. Gynecol Oncol. 2008;109:86-91.

15. Wang W, Chu HJ, Shang CL, Gong X, Liu TY, Zhao YH, et al. Long-term oncological outcomes after laparoscopic versus abdominal radical hysterectomy in stage IA2 to IIA2 cervical cancer: a matched cohort study. Int J Gynecol Cancer. 2016;26: 1264-73.

16. Soliman PT, Frumovitz M, Sun CC, Dos Reis R, Schmeler KM, Nick AM, et al. Radical hysterectomy: a comparison of surgical approaches after adoption of robotic surgery in gynecologic oncology. Gynecol Oncol. 2011;123:333-6.

17. Wright JD, Herzog TJ, Neugut AI, Burke WM, Lu YS, Lewin $\mathrm{SN}$, et al. Comparative effectiveness of minimally invasive and abdominal radical hysterectomy for cervical cancer. Gynecol Oncol. 2012;127:11-7.

18. Laparoscopic approach to cervical cancer (LACC) [Internet]. Bethesda, MD: U.S. National Library of Medicine; 2018 [cited 
2018 May 20]. Available from: https:// clinicaltrials.gov/.

19. World Health Organization. International statistical classification of diseases and related health problems, 10th rev. ed. Geneva: World Health Organization; 1994.

20. Korean Health Insurance Review and Assessment Service. Cost of health insurance medical care benefits. Seoul: Korean Health Insurance Review and Assessment Service; 2016.

21. Elixhauser A, Steiner C, Harris DR, Coffey RM. Comorbidity measures for use with administrative data. Med Care. 1998;36: 8-27.

22. Sohn S, Kim J, Chung CK, Lee NR, Park E, Chang UK, et al. A nationwide epidemiological study of newly diagnosed spine metastasis in the adult Korean population. Spine J. 2016;16: 937-45.

23. Sohn S, Kim J, Chung CK, Lee NR, Sohn MJ, Kim SH. A nation-wide epidemiological study of newly diagnosed primary spine tumor in the adult Korean population, 2009-2011. J Korean Neurosurg Soc. 2017;60:195-204.

24. Robins JM, Hernan MA, Brumback B. Marginal structural models and causal inference in epidemiology. Epidemiology. 2000;11:550-60.

25. Stukel TA, Fisher ES, Wennberg DE, Alter DA, Gottlieb DJ, Vermeulen MJ. Analysis of observational studies in the presence of treatment selection bias: effects of invasive cardiac management on AMI survival using propensity score and instrumental variable methods. JAMA. 2007;297:278-85.

26. Wright JD, Burke WM, Tergas AI, Hou JY, Huang Y, Hu JC, et al. Comparative effectiveness of minimally invasive hysterectomy for endometrial cancer. J Clin Oncol. 2016;34:1087-96.

27. Barnett JC, Judd JP, Wu JM, Scales CD Jr, Myers ER, Havrilesky LJ. Cost comparison among robotic, laparoscopic, and open hysterectomy for endometrial cancer. Obstet Gynecol. 2010; 116:685-93.

28. Rauh-Hain JA. Comparative effectiveness of minimally invasive staging surgery in women with early stage cervical cancer. In: Proceedings of the Society of Gynecologic Oncologists; 2018 Mar 24-27; New Orleans, LA.

29. Ramirez PT, Frumovitz M, Pareja R, Lopez A, Vieira MA, Ribeiro R. Phase III randomized trial of laparoscopic or robotic versus abdominal radical hysterectomy in patients with earlystage cervical cancer: LACC Trial. In: Proceedings of the 49th Annual Meeting of the Society of Gynecologic Oncologists; 2018 Mar 24-27; New Orleans, LA. Chicago, IL: Society of Gynecologic Oncology; 2018.

30. Park JY, Nam JH. How should gynecologic oncologists react to the unexpected results of LACC trial? J Gynecol Oncol. 2018;29:e74.

31. Kimmig R, Ind T. Minimally invasive surgery for cervical cancer: consequences for treatment after LACC Study. J Gynecol Oncol. 2018;29:e75.

32. Kanao H, Aoki Y, Takeshima N. Unexpected result of minimally invasive surgery for cervical cancer. J Gynecol Oncol. 2018;29:e73. 